\title{
Spectrum of findings in 99mTc-MAA SPECT/CT and their significance in treatment planning for Yttrium 90-microsphere radioembolization for hepatocellular carcinoma
}

\author{
Daniel T. Myers* \\ Department of Radiology, Henry Ford Hospital, Detroit, USA
}

\begin{abstract}
The primary utility of 99mTc-MAA SPECT/CT is to quantify lung shunt fractions and identify additional sites of extra-hepatic uptake in the pre-therapy evaluation for Yttrium-90-microsphere (Y90) radioembolization of the liver. After a review of 99mTc-MAA intra-arterial injection and SPECT/CT imaging technique, the evaluation of lung shunt fraction and it significance will be discussed. The potential morbidities from extra-hepatic distribution including radiation pneumonitis, gastrointestinal tract ulceration / bleeding and cholecystitis will be reviewed. Findings on 99mTc-MAA SPECT/CT that may preclude the patient from receiving radioembolization will be identified. Multiple examples of extra-hepatic 99mTc-MAA distribution involving gallbladder, duodenum, stomach, and portal vein tumor thrombus will be displayed with correlative fused and diagnostic CT imaging. The impact of extra-hepatic $99 \mathrm{mTc}-\mathrm{MAA}$ identified by the nuclear physician on the treatment plan will be reviewed. This will include the role of prophylactic arterial bland arterial embolization / coiling with correlative arteriograms. Examples of pitfalls in extra-hepatic uptake including $99 \mathrm{mTc}-\mathrm{MAA}$ breakdown products in thyroid, stomach and kidneys will be shown and potential technique changes to ameliorate these pitfalls will be discussed.
\end{abstract}

\section{Introduction}

Surgical percutaneous intervention and/or resection with the ultimate goal of transplantation for patients with primary hepatocellular carcinoma (HCC) remains the gold standard for treatment and represents an opportunity for cure; however, candidates for resection or transplantation represent the minority of patients presenting with HCC [1]. Even patients who meet the Milan criteria for liver transplantation may experience progression of disease as they wait for a matching donor. Locoregional approaches have emerged in these difficult patients whose comorbidities or clinical presentation exclude the option of surgery. Y90-microspheres (TheraSphere ${ }^{\star}$ ) has been shown to improve outcomes in patients with primary liver tumors [1]. It has the potential to downgrade patients who were initially outside the Milan criteria, so they may be candidates for transplant, and it offers palliation for patients with metastatic disease to the liver when first and second-line chemotherapy has failed [1].

HCC is typically a highly vascular tumor which can contain arteriovenous shunts, which bypass the capillary network. Patients with cirrhosis and portal hypertension may also have foci of arteriovenous shunting unrelated to malignancy. Thus, a concern of Y 90 -microsphere therapy is the potential for radioembolic material reaching the lungs. The MAA particles closely approximate the size and distribution of the Y 90 -microspheres. The mean diameter is $35 \mu \mathrm{m}$ with a range of 30 $90 \mu \mathrm{m}$. The Y90-microspheres are $15-35 \mu \mathrm{m}$ in diameter. The primary purpose of 99mTc-MAA SPECT/CT is to estimate the percent Y90microspheres that would be delivered to the lung so that an appropriate dose can be used, minimizing the possibility of radiation-induced pneumonitis. The second purpose is to identify additional sites of extra- hepatic uptake which have the potential to alter or even preclude the radioembolization procedure.

\section{Technique}

A thorough angiographic examination of the abdomen is required prior to radioembolization. The celiac artery, superior mesenteric artery (SMA), common and proper hepatic arteries, gastroduodenal artery (GDA), and right and left hepatic arteries are studied. Variant anatomy is noted including replaced or accessory hepatic arteries as well as the position of the GDA and right gastric artery.

The specific therapy planned will dictate the feeding vessel to be examined with 99mTc-MAA. For example, if a staged approach is used due to multifocal disease, only the artery that is to be radio embolized is infused with 99mTc-MAA. Different lobes and tumors each have the potential to yield different lung shunt fractions. Once the appropriate feeding artery has been selected, approximately $4 \mathrm{mCi}$ of $99 \mathrm{mTc}-\mathrm{MAA}$ particles are infused. If more than one artery is to be embolized at one setting, the dose of $99 \mathrm{mTc}-\mathrm{MAA}$ should be fractionated among the different arteries. The patient is then brought to the nuclear medicine department for imaging.

Many different imaging techniques have been utilized to identify sites of extrahepatic uptake including planar imaging, SPECT alone,

${ }^{\star}$ Correspondence to: Daniel T. Myers, Department of Radiology, Henry Ford Hospital, Detroit, MI 48202, USA, Tel: + 313916 1033; Email: danielm@rad.hfh.edu

Received: February 28, 2018; Accepted: March 22, 2018; Published: March 26, 2018 
and SPECT/CT. SPECT/CT has been shown to be more sensitive and accurate in defining sites of extrahepatic uptake [2]. CT allows anatomic localization and provides data for attenuation correction. Various software is available that will allow the nuclear physician to fuse the images. The lung shunt fraction is calculated by dividing the net counts in the lungs (obtained by geometric mean) by the sum of the net counts in the lungs and liver (Figure 1).

\section{Examples of extra-hepatic uptake}

Tumor embolization therapy carries an ever-present risk of complications due to nontarget embolization. This risk carries greater importance in cases of Y90-microsphere embolization because Y 90 is a pure beta emitter with a mean energy of $0.94 \mathrm{MeV}$ and mean tissue penetration of $2.5 \mathrm{~mm}$. Thus, the nuclear physician plays a critical role in the management of patients in preparation for Y90-microsphere embolization. Recognition of extrahepatic uptake of 99mTc-MAA can change the management and avoid serious complications.

A dose of greater than 30 Gy delivered to the lungs during a single infusion or 50 Gy cumulative dose is considered an absolute contraindication for Y90-microsphere therapy [3]. Some authors have advocated a lower dose threshold for patients with limited pulmonary reserve [4]. A recent article based on glass microspheres demonstrated no cases of radiation pneumonitis in patients receiving greater than recommended doses in 58 patients [5]. Although the calculated cumulative dose dictates the feasibility of performing Y 90 embolization, in general, a lung shunt fraction less than $20 \%$ is considered safe [6].

Radiotracer accumulation in the gallbladder has a reported incidence of $12 \%$ [7]. Although blood flow to the gallbladder is predominantly from the cystic artery which typically originates from the right hepatic artery, there is significant variation (Figure 2). The cystic artery may arise from the left hepatic, middle hepatic, GDA, or accessory arteries. Identification of radiotracer accumulation in the gallbladder can direct the operator to place the embolization catheter distal to the cystic artery. Occasionally, the cystic artery arises from a deep branch of the right hepatic artery, precluding this option; additionally, if the embolization catheter is not sufficiently proximal in the desired artery, a nonuniform distribution of Y90-microspheres may be delivered producing preferential flow to a segment. Prophylactic coil embolization of the cystic artery is commonly done to avoid this complication [6]. Prophylactic embolization carries a low risk of ischemic cholecystitis because of the presence of collateral perforating

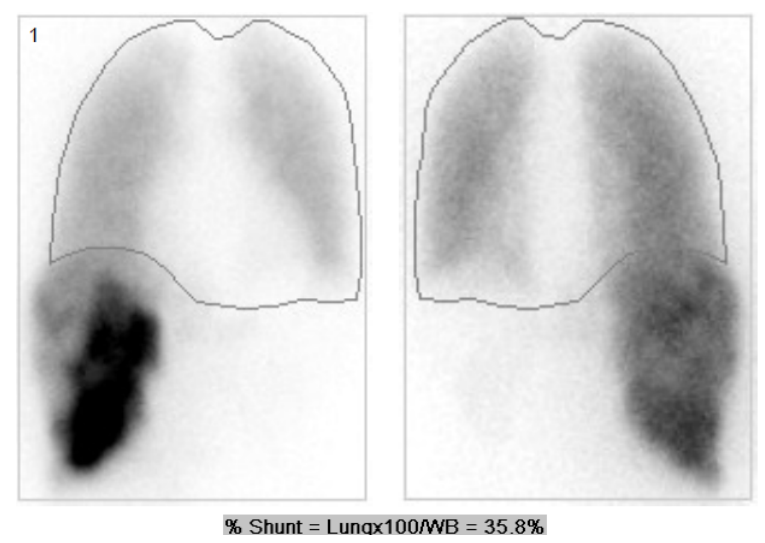

Figure 1. Anterior and posterior planar 99mTc-MAA images shows significant extrahepatic uptake in the lungs. Regions of interest were drawn around the whole body and lungs. The lung shunt fraction in this patient was $36 \%$. Planned therapy was cancelled due to an estimated dose of 92 Gy to the lungs.
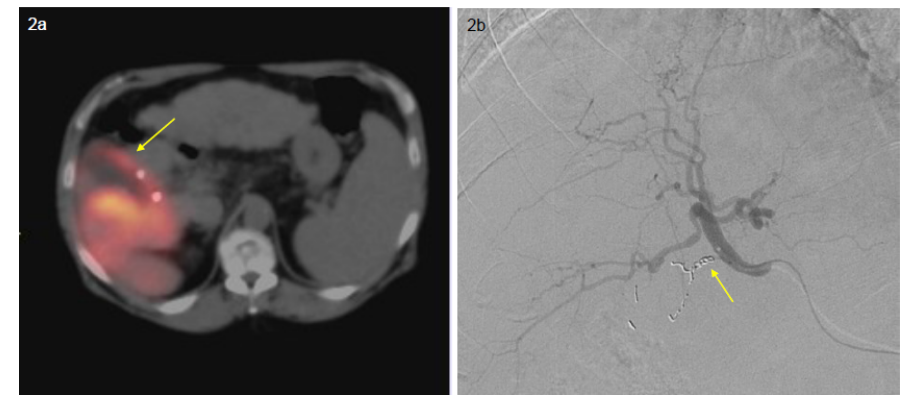

Figure 2a, 2b. SPECT/CT shows radiotracer uptake in the wall of the gallbladder despite prophylactic coil embolization, likely due to col-lateral vessels 2a. DSA shows coils in the cystic artery.

2b. Post-radioembolization CT (not shown) was performed showing no evidence of cholecystitis.

vessels from the liver; however, cases of ischemic cholecystitis are usually self-limited with supportive care the only necessary treatment.

Accumulation of $99 \mathrm{mTc}-\mathrm{MAA}$ in the GI tract is another commonly encountered scenario. This may manifest with uptake of $99 \mathrm{mTc}-$ MAA in the stomach, duodenum, or more distal intestines (Figure 3). True extrahepatic uptake of 99 mTc-MAA in the stomach must be differentiated from radiotracer activity from free $99 \mathrm{mTc}$-pertechnetate. Non-target embolization of the GI tract with Y90-microspheres may result in gastritis/enteritis or ulceration. Ulcers secondary to radioembolization differ from peptic ulcers. Radioembolic ulcers arise from the serosal surface. This may impair normal wound healing and can make the ulcers endoscopically occult [6]. Accumulation of $99 \mathrm{mTc}$ MAA in the duodenum is often best recognized on coronal SPECT [7]. Because of the risk of radioembolic material refluxing into the arteries supplying the stomach and intestines, many operators prophylactically coil embolize the GDA and the right gastric artery.

Nontarget embolization of the anterior abdominal wall is another recognized risk of embolization. This can occur by inadvertent embolization of the hepatic falciform artery. The patency of this artery is variable; it most frequently arises from the middle or left hepatic artery. The reported incidence of detecting radiotracer activity in the falciform ligament is $10 \%$. On SPECT/CT, the radiotracer activity appears in a linear distribution and extends from the anterior aspect of the liver inferiorly towards the umbilicus (Figure 4). Uptake is usually faint and may require adjustment of image intensity to be clearly seen [8].

Main portal vein thrombosis (PVT) is considered a relative contraindication to radioembolization. In contrast to chemo- or bland embolization; Y90 radioembolization exerts less of an ischemic effect due to the size of the particles. There may be an increasing role of radioembolization in the future in the presence of PVT as recent evidence suggests that it may prolong survival in patients with HCC and PVT [9]. Because the intravascular tumor is still supplied by the hepatic artery, SPECT/CT will demonstrate uptake within the portal vein (Figure 5).

\section{Imaging pitfalls}

Small differences in the properties of 99mTc-MAA and Y90microspheres can account for overestimation of the degree of lung shunting. One important difference is related to the substrate to which the radioactive particle binds. As opposed to the glass particles which constitute Y90-microspheres, MAA is composed of protein. If there is delay between infusion of $99 \mathrm{mTc}-\mathrm{MAA}$ and imaging, a significant amount of MAA may be broken down, allowing $99 \mathrm{mTc}$ to pass through 

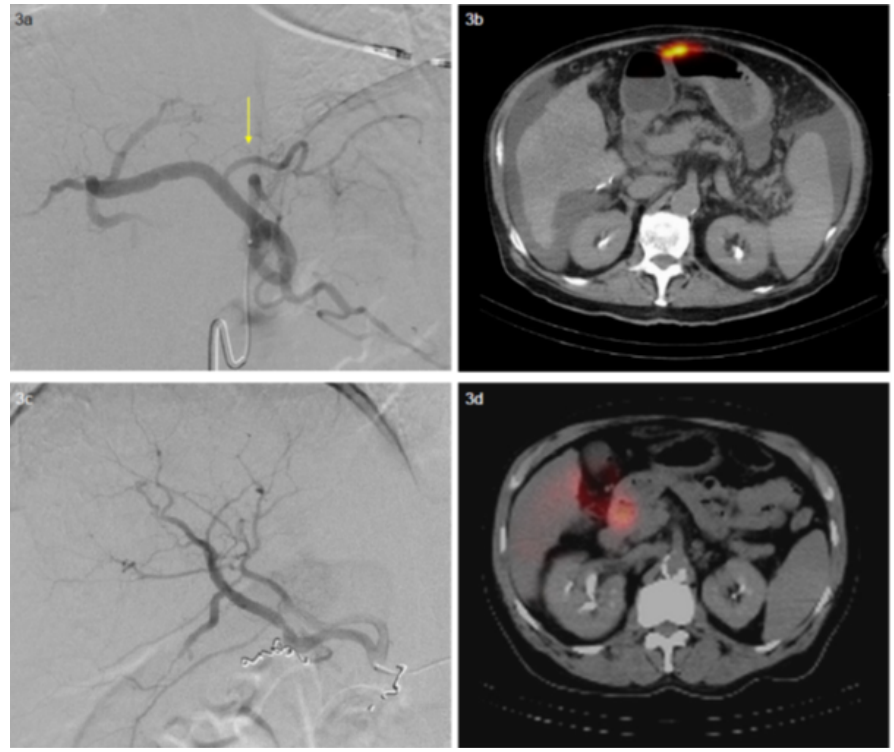

Figure 3a, 3b, 3c, 3d:

3a. Angiogram of a branch of the celiac artery shows a common gastrohepatic trunk. 3b. SPECT/CT demonstrates radiotracer uptake along the anterior wall of the stomach despite prophylactic coil embolization (not shown).

3c. Angiogram of the right hepatic artery shows early bifurcation and coils within a duodenal artery branch.

3d. SPECT/CT demonstrates radiotracer uptake within the descending duodenum. Due to persistent GI activity, chemoembolization was performed for this patient.
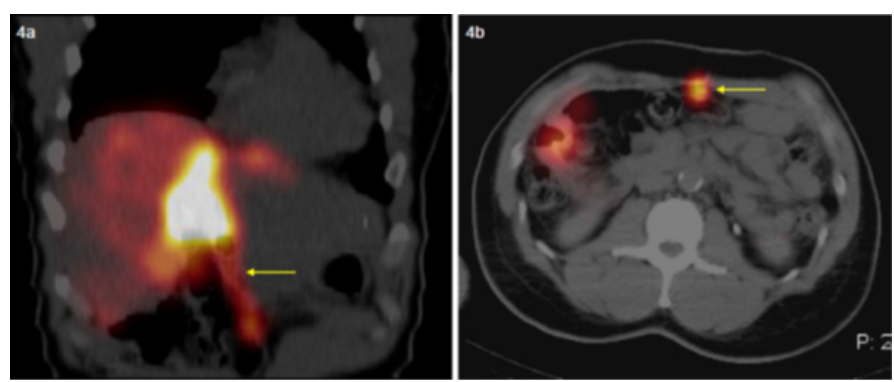

Figure 4a, 4b. SPECT/CT images demonstrate linear uptake directed towards the umbilicus, within the falciform artery.

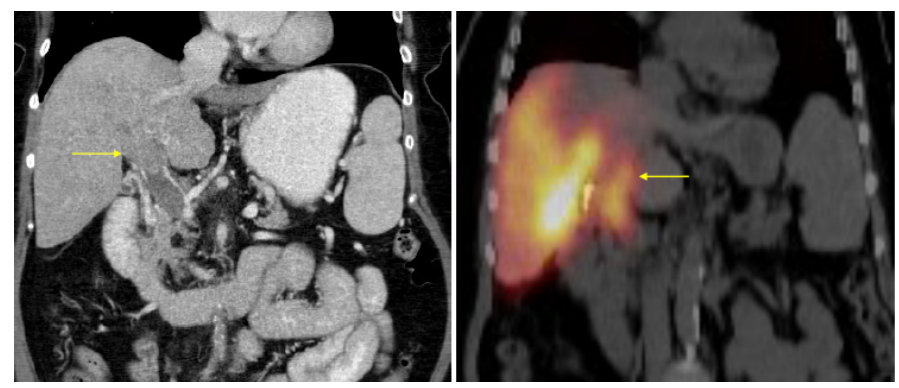

Figure 5a, 5b. CT demonstrates tumor in the portal vein, SPECT/CT shows radiotracer uptake within the tumor thrombus.

the capillary bed and into the lung [4]. It is recommended that patients be transferred to the nuclear medicine department as quickly as possible after infusion to prevent this problem.

Additionally, although MAA particles are generally between 30-90 $\mu \mathrm{m}$, a small percentage will be smaller than $10 \mu \mathrm{m}$, allowing shunting through the capillary network. Most manufacturers state that fewer than $10 \%$ of MAA particles will be smaller than $10 \mu \mathrm{m}$. $95 \%$ of $\mathrm{Y} 90$ microspheres are between $15-35 \mu \mathrm{m}$ in diameter [6].
Free technetium will manifest as uptake in the stomach, salivary glands, thyroid gland, and kidneys (Figure 6). If this constellation of findings is seen, this can be interpreted as free technetium. However, if there is isolated uptake in the gastric mucosa, this may represent true extrahepatic shunting and a search for a feeding vessel should commence.

Perchlorate has been shown to reduce uptake of free technetium in the thyroid and gastric mucosa. This is accomplished through competitive inhibition of technetium by the perchlorate ion preventing binding in the thyroid and stomach. Many institutions, including ours, administer $600 \mathrm{mg}$ perchlorate orally 30-60 minutes prior to the start of the 99mTc-MAA intra-arterial injection [10].

\section{Conclusion}

The utilization of Y90-microspheres for the treatment of HCC continues to increase. SPECT/CT has supplanted conventional SPECT imaging with its superior localization of extrahepatic uptake. The nuclear medicine physician plays a critical role in the interpretation of the pre-embolization 99mTc-MAA SPECT/CT image. The primary use of the study is to quantify the lung shunt fraction and identify additional sites of extra-hepatic radiotracer uptake. Common sites of extra-hepatic uptake include the gallbladder, stomach, and small bowel. More uncommon examples include falciform artery uptake and uptake within a portal vein thrombus. The recognition of these findings is important for treatment planning and for the prevention of nontarget embolization. The $99 \mathrm{mTc}-\mathrm{MAA}$ study is susceptible to certain pitfalls including free technetium and artifacts related to differences in MAA and glass particles. Careful adherence to the imaging protocol and prophylactic medication can help ameliorate these difficulties.

\section{6}

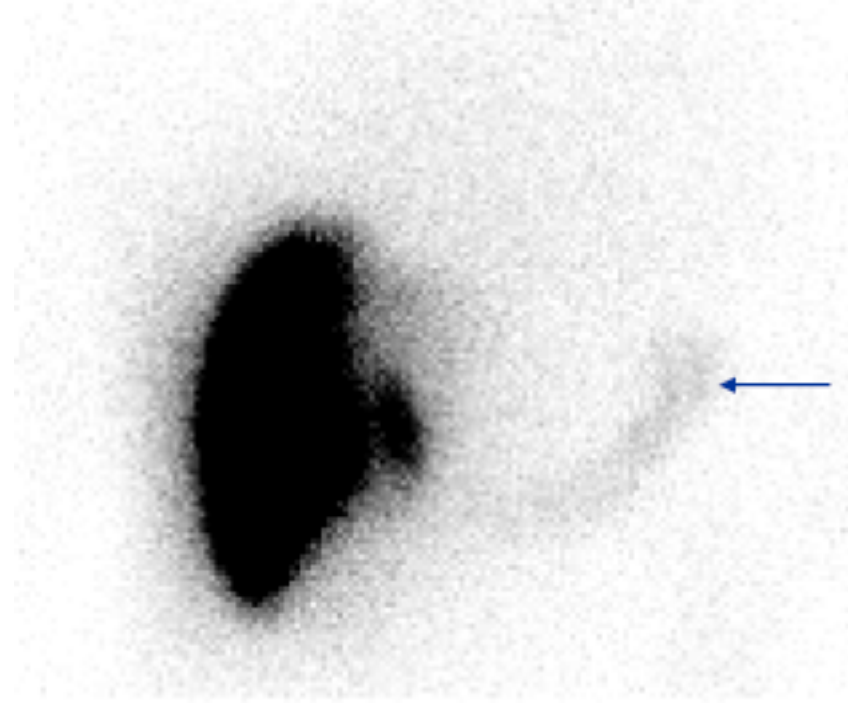

Figure 6. Planar 99mTc-MAA image shows uptake in the thyroid and stomach, consistent with free technetium 


\section{References}

1. Riaz A, Lewandowski RJ, Kulik L, Salem R (2009) Yttrium-90 radioembolization using TheraSphere in the management of primary and secondary liver tumors. Q J Nucl Med Mol Imaging 53: 311-316. [Crossref]

2. Hamami ME, Poeppel TD, Müller S, Heusner T, Bockisch A, et al. (2009) SPECT/CT with $99 \mathrm{mTc}-\mathrm{MAA}$ in radioembolization with Y90 microspheres in patients with $\mathrm{HCC}$ J Nucl Med 50: 688-692. [Crossref]

3. Ho S, Lau WY, Leung TW, Chan M, Johnson PJ, et al. (1997) Clinical evaluation of the partition model for estimating radiation doses from yttrium-90 microspheres in the treatment of hepatic cancer. Eur J Nucl Med 24: 293-298. [Crossref]

4. Salem R, Thurston KG (2006) Radioembolization with Y90 microspheres: a state-ofthe-art brachytherapy treatment for primary and secondary liver malignancies. Part 1: Technical and methodologic considerations. J Vasc Interv Radiol 17: 1251-1278. [Crossref]

5. Salem R, Parikh P, Atassi B, Lewandowski RJ, Ryu RK, et al. (2008) Incidence of radiation pneumonitis after hepatic intra-arterial radiotherapy with Y 90 microspheres assuming uniform lung distribution. Am J Clin Oncol 31: 431-438. [Crossref]
6. Lewandowski RJ, Sato KT, Atassi B, Ryu RK, Nemcek AA Jr, et al. (2007) Radioembolization with Y 90 microspheres: angiographic and technical considerations. Cardiovasc Intervent Radiol 30: 571-592. [Crossref]

7. Ahmadzadehfar H, Sabet A, Biermann K, Muckle M, Brockmann H, et al. (2010) The significance of 99mTc-MAA SPECT/CT liver perfusion imaging in treatment planning for Y90-microsphere selective internal radiation treatment. J Nucl Med 51: 1206-1212. [Crossref]

8. Kao YH, Tan AE, Khoo LS, Lo RH, Chow PK, et al. (2011) Hepatic falciform ligament Tc-99m-MAA activity on SPECT/CT prior to Y90 microsphere radioembolization: prophylactic measures to prevent non-target microsphere localization via patent hepatic falciform arteries. Ann Nucl Med 25: 365-369. [Crossref]

9. Tsai, Burke CT, Kennedy AS, Moore DT, Mauro MA, et al. (2010) Use of Yttrium-90 microspheres in patients with advanced HCC and portal vein thrombosis. $J$ Vasc Interv Radiol 21: 1377-1384. [Crossref]

10. Oldendorf WH, Sisson WB, Iisaka Y (1970) Compartmental redistribution of 99mTcPertechnetate in the presence of perchlorate ion and its relation to plasma protein binding. J Nuc Med 11: 85-88. [Crossref]

Copyright: $(02018$ Myers DT. This is an open-access article distributed under the terms of the Creative Commons Attribution License, which permits unrestricted use, distribution, and reproduction in any medium, provided the original author and source are credited. 